\title{
Estudiantes extranjeros en Santa Fe. El caso de las cohortes brasileñas del Doctorado de Educación de la UCSF
}

\author{
Foreign students in Santa Fe. The case of the Brazilian \\ cohorts of the UCSF Doctorate of Education
}

\section{Estudantes estrangeiros em Santa Fe. O caso das coortes brasileiras no Doutorado de Educação da UCSF}

\author{
Anabel Gaitán \\ Doctora en Educación \\ Universidad Católica de Santa Fe \\ agaitan@ucsf.edu.ar \\ María Gabriela Pauli \\ Doctora en Educación \\ Universidad Católica de Santa Fe \\ gpauli@ucsf.edu.ar
}

\section{Resumen}

La propuesta de abordar la migración académica Sur-Sur en educación superior en el presente siglo, nos ha invitado a analizar una experiencia como ha sido la de las cohortes de doctorandos provenientes de Brasil en la Universidad Católica de Santa Fe entre 2006 y 2013, 17 cohortes en total. Se trata de una experiencia particular porque estas cohortes se conformaron exclusivamente con estudiantes brasileños, salvo una que estuvo integrada también por un subgrupo de angoleños.

Metodológicamente nos proponemos abordar el caso desde dos perspectivas; por un lado, las decisiones en el marco de la política de la Universidad que hizo posible esta experiencia, y por otro lado, el análisis de las subjetividades de sus actores, especialmente de los doctorandos a partir de la interpretación de la consulta directa realizada a los estudiantes.

La discusión final está dada por la evaluación de la experiencia y la cosecha que tanto alumnos como instituciones recogen a través del tiempo.

Palabras clave: Educación, posgrado, movilidad académica, subjetividades, UCSF. 


\section{Summary}

The proposal to address South-South academic migration in higher education in this century has invited us to analyze an experience such as that of the cohorts of doctoral students from Brazil at the Catholic University of Santa Fe between 2006 and 2013, 17 cohorts in total. This is a particular experience because these cohorts were made up exclusively of Brazilian students, except for one that was also made up of a subgroup of Angolans.

Methodologically, we propose to approach the case from two perspectives; on the one hand, the decisions within the framework of the University's policy that made this experience possible, and on the other hand, the analysis of the subjectivities of its actors, especially the doctoral students, based on the interpretation of the direct consultation made to the students.

The final discussion is given by the evaluation of the experience and the harvest that both students and institutions gather over time.

Keywords: Education, postgraduate, academic mobility, subjectivities, UCSF.

\section{Resumo}

A proposta de abordagem da migração acadêmica Sul-Sul em educação superior no século presente tem nos levado a analisar a experiência das coortes de doutorandos oriundos do Brasil na Universidade Católica de Santa Fe entre 2006 e 2013, para um total de 17 coortes. Trata-se de uma experiencia particular porque essas coortes se conformaram exclusivamente por estudantes brasileiros, salvo uma que esteve integrada também por um subgrupo de angolanos.

Metodologicamente, propõe-se abordar esse caso desde duas perspectivas; de um lado, a través das decisões no marco da política da Universidade que fez possível essa experiência, por outro lado, a análise das subjetividades dos seus atores, especialmente dos doutorandos a partir das interpretações da consulta direta realizada aos estudantes.

A discussão final está focada na avaliação da experiencia e a colheita que tanto os alunos como as instituições colhem a través do tempo.

Palavras-chave: Educação, pós-graduação, mobilidade académica, subjetividades, UCSF.

\section{Introducción}

Las migraciones académicas en el ámbito de la educación superior constituyen un caso particular del fenómeno de la movilidad poblacional, tanto por las razones que las mueven como por el perfil de los migrantes y la duración de la estadía en los países receptores.

En las últimas décadas se ha incrementado la movilidad entre países de América del Sur, en virtud de una serie de factores, entre los que resulta posible destacar las políticas de cooperación internacional que desde el campo de los acuerdos políticos y económicos se extendieron al educativo y científico-tecnológico. El MERCOSUR, por ejemplo, produjo una serie de acuerdos y estrategias comunes a los países miembros que favorecieron los contactos e intercambios entre instituciones educativas de nivel superior. 
Nos interesa, en el presente artículo detenernos en un proceso en particular, que se produjo en Santa Fe, Argentina, entre 2006 y 2014. En ese lapso, la Universidad Católica de Santa Fe, abrió diez cohortes de su Doctorado en Educación, y siete del Doctorado en Derecho, destinadas exclusivamente a estudiantes extranjeros en el marco de una movilidad sur-sur, debido a la recepción direccionada de estudiantes del país vecino, Brasil.

Desde luego, este proceso regional se inscribe en el contexto de las migraciones a Argentina, un país receptor por excelencia de estudiantes extranjeros que lo eligen para cursar sus estudios de grado $^{1}$ o de posgrado. El prestigio de sus universidades, las facilidades del ingreso irrestricto, los costos de estudiar en Argentina parecen ser razones que convierten al país austral en un destino apetecible para estudiantes extranjeros.

El caso del que nos ocuparemos a continuación es muy particular, ya que se trata de cohortes donde todos los doctorandos fueron extranjeros, en 15 de ellas brasileños, en una además de brasileños se incorporaron dos doctorandos mexicanos y dos ecuatorianos, y en una se sumaron unos pocos estudiantes procedentes de Angola. La experiencia de estas cohortes puras de extranjeros en la UCSF resulta especialmente interesante por ser original.

Nos proponemos indagar en torno a ella, analizando desde una perspectiva de política educativa, las decisiones de la Casa de Estudios que hicieron posible la experiencia; y desde la perspectiva de las subjetividades en juego, el impacto en los sujetos - las personas - que fueron parte de ella.
Si bien la bibliografía sobre el tema es copiosa, no es demasiado lo que se ha indagado acerca de estos fenómenos en la ciudad de Santa Fe Argentina, y de hecho, no hay investigaciones que den cuenta de la presencia de estudiantes extranjeros en la Universidad Católica de Santa Fe (en adelante UCSF) ${ }^{2}$.

\section{Marco teórico}

Referirnos a migraciones académicas, exige precisar el concepto mismo de migración y el de migración académica, así como revisar otras categorías analíticas que también se utilizan para abordar temas vinculados a la movilidad y las relaciones que se generan entre distintas universidades o instituciones de educación superior, como son las de cooperación internacional, internacionalización, o movilidad académica.

Para ello, hemos decidido tomar artículos recientes que nos permitan definir conceptos tales como el de migraciones, movilidad académica o internacionalización y luego contrastar con la experiencia que se llevó a cabo en la UCSF entre 2006 y 2013 para poder determinar cuál es el marco conceptual más apropiado para abordar su estudio. Si bien el caso a estudiar es un ciclo de cohortes que se cerró en 2013, nuestro análisis es contemporáneo y se inscribe en la línea de las producciones actuales sobre el tema.

Entre los trabajos que se ocupan de estudiantes extranjeros en Argentina, Sangiácomo analiza el caso de estudiantes bolivianos en la Universidad de La Plata, y en su análisis, delimita la condición de migrante de acuerdo con la legislación del país y sostiene que

\footnotetext{
${ }^{1}$ En Argentina, usualmente se denominan estudios de grado a aquellos destinados a la obtención de un título de grado. Es equivalente a estudios de pregrado en otros contextos.

${ }^{2}$ La ciudad de Santa Fe ha contado históricamente con tres universidades, dos de ellas nacionales y de gestión pública: la Universidad del Litoral y la Universidad Tecnológica - Facultad Regional Santa Fe; y una universidad privada, la UCSF, que es nuestro objeto de estudio.
}

PAIDEIA, No. 25. Universidad Surcolombiana / Facultad de Educación, 2020 
"La migración de estudiantes se define por su carácter temporal determinado por la duración de las carreras, así como también por el status legal con el que permanecen en nuestro país mientras realizan esta actividad". (2002, p. 206)

Es decir que las variables de residencia y status legal definen según la autora la condición de migrante. Por su parte, Cardozo, en una investigación más reciente acerca del fenómeno en la Universidad de Lanús, sostiene que son estudiantes extranjeros aquellos que no tienen nacionalidad argentina, sea se han radicado en Argentina desde tiempo atrás y deciden comenzar o continuar estudios en el país, o que se trasladen solo para cursar una carrera.

La autora clasifica a los estudiantes extranjeros haciendo la distinción entre migrantes permanentes, que son aquellos cuyo título anterior ha sido otorgado por una institución argentina y estudiantes internacionales, que son los que poseen un título anterior extranjero. Subdivide a estos últimos en migrantes permanentes y no permanentes, según deseen radicarse o no en el país, y establece una categoría de no migrante que engloba a quienes "no cambian su residencia para estudiar, utilizan la visa de turista y vienen a Argentina en los períodos de cursada. Suelen participar de las modalidades semipresenciales o a distancia, aunque también de las presenciales." (Cardozo, 2015, p 2)

También Sosa adopta el concepto de migrantes en $\mathrm{su}$ trabajo referido al caso argentino, y explicita el marco político de este proceso que se inscribe en los acuerdos del MERCOSUR, cuya agenda, sostiene "[,,.] estuvo desde el comienzo fuertemente concentrada en los temas de educación superior, que incluye la enseñanza universitaria y de posgrado". (2016 p 102)

Describiendo las acciones que se propiciaron, alude a la movilidad académica regional, que está favorecida por estrategias como son la acreditación de títulos de grado, y "[...] la creación de un espacio regional común en educación superior por medio de acciones de gestión académica e institucional, de la movilidad de estudiantes, de transferencia de créditos y de intercambio de docentes e investigadores[...]" (Sosa, 2016, p. 103) En este marco menciona programas como el de Acreditación Regional de Carreras Universitarias del Sur (ARCU-SUR) y el programa MARCA. Se trata de políticas destinadas a homogeneizar los criterios de acreditación de carreras y títulos, estableciendo estándares comunes a los países que suscriben los acuerdos, y sistemas de evaluación de la calidad educativa.

Hasta aquí hemos hecho referencia a estudios que refieren a la temática en diversos casos de Argentina, tomando el concepto de migración o migración académica, pero es copiosa la bibliografía que remite al mismo fenómeno con el concepto de internacionalización. Quienes prefieren hablar de migración focalizan su análisis en las subjetividades de los estudiantes que se movilizan para estudiar en otros países, las dificultades que afrontan, sus percepciones y vivencias; en cambio, los autores que eligen el concepto de internacionalización ponen el acento en los acuerdos y las políticas para favorecer la movilidad de estudiantes y de profesores, así como las actividades conjuntas entre casas de estudio de diferentes países.

Es mucho más nutrida la producción académica que analiza el fenómeno de la movilidad académica internacional en términos de internacionalización. Por ejemplo, en un trabajo de reciente publicación, Ramírez Valdivia y Latorre Bahamondez, destacan la importancia de los programas supranacionales como el caso de ERASMUS y el Proceso de Bolonia para Europa y sostienen que ellos constituyeron los primeros eslabones de una nueva concepción sobre el tema:

Desde los años 90, la internacionalización se vuelve más compleja y se asocia con la globalización, educación transnacional y 
redes internacionales. Es decir, se asocia a un mundo interconectado y más uniforme, donde confluyen las diversas culturas. (Ramirez Valdivia y Latorre Bahamondez, 2019 , p. 52)

Autores como Lamfri y Salto (2016), o Abba (2019) inscriben también el discurso sobre internacionalización en términos de cooperación académicas. Abba, por su parte, reseña cuatro publicaciones sobre las políticas y estrategias de internacionalización de la educación en el contexto latinoamericano. En ellas participaron gestores de las políticas, convocados en dos casos por universidades argentinas: la Universidad Nacional de General Sarmiento en 2013 y posteriormente la Universidad de Lanús. Los otros dos fueron elaborados por las investigadoras Daniela Perrota y María Isabel da Cunha. (Abba, 2019, p. 85)

Explica Abba que, con la asunción de gobiernos progresistas en varios países latinoamericanos a comienzos del siglo XXI, se generó un contexto propicio para el desarrollo de la internacionalización académica. Ello se plasmó en documentos como la Declaración de la Conferencia Regional de Educación Superior, en Cartagena de Indias en el año 2008, que, atendiendo a una perspectiva de internacionalización solidaria, promovía la cooperación sur-sur y la integración regional. Para ello favorecía la creación de espacios académicos comunes entre universidades de distintos países de la región. Se observa entonces, que este concepto de internacionalización solidaria excede ampliamente la movilidad de estudiantes y profesores.

De las formulaciones sobre internacionalización de la educación, nos interesan especialmente dos. El de Passerini, Zucarelli y De León (2018) que enfatizan en el rol de las movilidades en los procesos de internacionalización académicas. Sostienen los autores que:

La internacionalización en la Educación Superior representa cada vez más una oportunidad para la mejora de la calidad de la formación de estudiantes y profesores. Una forma en la que la mayoría de las Universidades promueven esta función es a través de movilidades, generalmente con intercambios, enviando y recibiendo alumnos y docentes, financiando de forma parcial o total los gastos en los que se incurre en los traslados, alojamiento y alimentación de las personas involucradas. (p 119)

Ellos analizan comparativa-mente el caso de cuatro universidades del cono sur, la UNL (Argentina), la UNESP y la UNA (Brasil) y la Udelar (Uruguay) desde una perspectiva centrada en las experiencias y expectativas de los sujetos que se mueven para estudiar.

El otro trabajo que aporta a la precisión conceptual de nuestra investigación es el de Mayer y Catalano (2018) quienes también vinculan estrechamente internacionalización y movilidad.

El movimiento físico de las personas que implica un origen $y$ un destino acarrea un conjunto de fenómenos que circulan satelitalmente sobre los sujetos, sus interacciones y significados, perceptible desde el nivel individual y también desde a escala social. Sostienen los autores que cuando el desplazamiento de las personas se produce con el motivo de cursar estudios de especialización o que completen su formación inicial, y se acota a un período, estamos en presencia de lo que definen como "movilidad por estudio" (Mayer y Catalano, 2018, p.29). Este movimiento favorece la generación de intercambios y de un espacio común entre las instituciones dedicadas a la educación superior.

Este breve recorrido teórico, nos posibilita una primera precisión conceptual. Tanto los autores que trabajan con el concepto de migración, como los que prefieren el de internacionalización, vinculan sus análisis al concepto de movilidad internacional, aun 
cuando apelan a distintos modos de nombrarlo: movilidad por estudio, movilidad a secas, movilidad académica, movimientos, entre otros.

Resulta pertinente para nuestra indagación adoptar el concepto de movilidad académica internacional, concepto que es lo suficientemente acotado para evitar ambigüedades y a la vez contiene los elementos que caracterizan al caso que estudiamos: estudiantes extranjeros (latinoamericanos y africanos) que se movilizan para estudiar su carrera de posgrado en la UCSF.

\section{Metodología}

Adoptar el concepto de movilidad académica internacional, nos permite a su vez, recuperar la dimensión de las subjetividades en que focalizan los estudios sobre migraciones académicas, y la dimensión de las políticas con relación al asunto que es el eje de los análisis sobre internacionalización de la educación.

Conviene para terminar este apartado, explicitar las decisiones metodológicas que hemos tomado. En primer lugar, decimos que el trabajo corresponde a un estudio de caso, el de las 17 cohortes de estudiantes extranjeros de los Doctorados de Educación y Derecho de la UCSF entre 2006 y 2013. Optamos por una metodología cualitativa analítico-hermenéutica, partiendo de la descripción de la experiencia y recuperando la valoración que hacen de ella los doctorandos. De ese modo, es posible trabajar con información cuantitativa y cualitativa en orden a poder evaluar la relevancia de la experiencia para las personas involucradas y para la Casa de Estudios. Apelamos a un enfoque exploratorio, que nos permita aproximarnos al caso en estudio y para ello diseñamos una encuesta semiestructurada que permite relevar datos e impresiones de los doctorandos. Obtuvimos 61 respuestas.

Los datos obtenidos de la aplicación de la encuesta se triangulan además con información acerca de las trayectorias académicas de los estudiantes que proporciona la base de datos de la Dirección de Posgrado de la Universidad. De este modo, es posible objetivar la información que proporcionan las encuestas.

\section{a. Las políticas educativas de la UCSF en relación con la movilidad académica internacional}

Hacia fines de la década de 1950 se inicia una etapa de creación de Universidades Privadas a partir del decreto ley $\mathrm{N}^{\circ} 6403$ de Organización de Universidades Nacionales, del 23 de diciembre de 1955. El 30 de septiembre de 1958 se sancionó una primera ley 14.557 de Universidades Privadas y el 29 de diciembre de 1967 la sanción de la Ley $\mathrm{N}^{\circ} 17604$ de Universidades Privadas, completaba el marco legal que favorecía la creación o restructuración de las casas de estudio. Surgen así la Universidad Católica de Córdoba, la Universidad Católica Argentina, la Universidad del Salvador, y la Universidad Católica de Santa Fe.

El cardenal Nicolás Fasolino, arzobispo de Santa Fe, propició la creación de la Universidad Católica promulgando, el 9 de junio de 1957, el Auto de Fundación de Instituto Libre ProUniversidad Católica de Santa Fe. El 15 de agosto de 1960, el Poder Ejecutivo de la Nación, expidió el Decreto $N^{\circ} 9621$ que reconocía la personería universitaria de la institución y autorizaba la expedición de títulos y diplomas. Nacía la UCSF.

La institución, inscribe la formación de sus estudiantes en los lineamientos que proporciona la Iglesia Católica a través de sus documentos, y se propone ofrecer un saber que

podría caracterizarse como "integral" no sólo porque refiere a diferentes campos, sino porque tiende a involucrar a la persona hasta el punto de movilizarlo en una búsqueda de toda la vida. El origen de su relación con el mundo y con la realidad será tendencialmente un juicio personal nacido 
de la pregunta por el sentido mismo y de las realidades en las que se actúa. Notemos que lo que puede mover a este tipo de iniciativa es la búsqueda de la verdad, como presupuesto que orienta el conocimiento posible tanto como el reconocimiento de su valor y de sus límites. (UCSF, 2014, p.19)

La organización de sus Facultades y la progresiva incorporación de carreras, el desarrollo de las áreas de investigación y extensión bajo diferentes modalidades, así como la construcción del edificio propio, demandan ingentes esfuerzos y algunos cuantos años.

Más adelante, surgieron las carreras de posgrado. De la oferta de la Universidad en este campo, nos interesa considerar especialmente el caso de los Doctorados en Educación y en Derecho, por ser las carreras que sostuvieron la experiencia de movilidad sur-sur que nos interesa estudiar. El Doctorado en Educación nace en el año 1996 y se mantiene vigente, habiendo pasado por sucesivas actuaciones del Ministerio de Educación de la Nación al darle reconocimiento oficial y validez nacional a tu título y por la Comisión Nacional de Evaluación y Acreditación Universitaria (CONEAU), acreditándolo en 1997, 2007 y 2017 respectivamente. El Doctorado en Derecho nace en el año 2004 y con modificaciones se mantiene vigente. Su denominación inicial fue modificada por la actual, Doctorado en Ciencia Jurídica. Este doctorado también ha sido reconocido y validado por el Ministerio de Educación de la Nación y recientemente acreditado por primera vez por la CONEAU a mediados de 2019 .

Si revisamos la copiosa producción académica que se ocupa de estudiar la movilidad en el caso de los estudios de posgrado y en particular los movimientos sur - sur, encontramos que los trabajos que se refieren a las Universidades argentinas focalizan en las de gestión pública. Es notoria la ausencia de estudios sobre el fenómeno de la movilidad académica internacional sur- sur en Universidades de gestión privada.
Aun así, podemos extraer algunas precisiones que sirvan para situar en contexto la experiencia de la UCSF. Los estudios de posgrado se desarrollaron exponencialmente en Argentina desde fines de la década de 1980, pero sin planificación. Ello ocasionó que se conformara como un "sistema desarticulado y diverso en cuanto a calidad y estructura" (García, 2016, p. 93), sin financiamiento público sistemático, lo que determinó que fueran los estudiantes quienes se hicieron cargo del costo de sus estudios de posgrado.

Aludiendo a esa situación, la misma autora sostiene que este proceso se dio en un contexto "[...] caracterizado por el desplazamiento de las decisiones institucionales desde el ámbito estatal y académico hacia el mercado." (García, 2016, p. 77) Esto explicaría tal vez la desarticulación de la oferta de posgrados. Al ser producto de demandas externas a los intereses de las Casas de Estudio, no constituyeron en los comienzos una política, sino que fueron surgiendo como respuesta a exigencias del mercado (entendiendo mercado tanto en términos educativos como económicos).

Los estudios de posgrado constituyen el principal factor de movilidad académica, según afirman Lampri y Salto (2016), y señalan que: "Lenta y paulatinamente se va incorporando la promoción de redes de investigadores y de instituciones que constituyen una potente estrategia de consolidación de equipos de investigación en articulación con la formación de posgrado. (p. 225)

Es decir, que serán ellos los que propicien el espacio para otras estrategias de cooperación académica internacional, como las redes y equipos de investigación que integren a las Universidades.

Lvovich (2009) afirma que el sistema de posgrados en Argentina es sumamente heterogéneo, disperso y con ofertas en ocasiones superpuestas; entendemos que esto es resultado 
de ese proceso de desarrollo que respondió a intereses y necesidades externas y no a una dinámica propia de las políticas universitarias estatales o institucionales.

El historiador señala que esa heterogeneidad y desarticulación es producto de la autonomía de las universidades argentinas, rasgo considerado muy valioso para el sistema educativo en este país. Como alternativa, afirma, la CONEAU ha diseñado un sistema de acreditación de carreras para las Universidades argentinas, que garantiza una cierta homogeneidad y una clasificación de niveles en la oferta de posgrados. Esta política, sumadas a otras similares de los países del MERCOSUR, derivaron posteriormente en la creación y puesta en marcha del sistema ARCU.SUR.

Este sistema exige de las Universidades un compromiso "[...] en programas de mejora continua de la calidad" (CONEAU, 2019, p. 12) y posibilita un mayor movimiento entre Casas de Estudios de países asociados al MERCOSUR, ya que la estandarización de los criterios de evaluación de la calidad académica posibilita el reconocimiento internacional de las titulaciones, el flujo de personas -profesores y estudiantesentre Universidades, y la creación de programas conjuntos.

Estos acuerdos y programas conformaron el marco en el que se desarrolló la experiencia de la que nos ocupamos a continuación.

b. El caso de los doctorandos de Brasil

Como ya hemos mencionado, las dos carreras -el Doctorado en Educación y el Doctorado en Derecho- ya eran parte de la oferta académica de la Universidad, cuando se tomó la decisión de recibir estas cohortes puras de extranjeros.

La experiencia no surgió de una decisión política de la UCSF, sino de la demanda de organizaciones que actuaban como intermediarias entre los interesados y diversas universidades de América del Sur.

Dos de estos organismos IFI (Instituto Faculdades Integradas) y ESJUS (Escola Superior de Justica), ambos radicados en Brasil, gestionaron la llegada de las cohortes de doctorandos. La vinculación se efectivizó a través de la Dirección de Posgrado de la Universidad y los convenios se firmaron con las autoridades de la Casa de Estudios. De este modo se dio lugar a cohortes cerradas de estudiantes extranjeros -en su mayoría brasileños.

Fueron 7 las cohortes que cursaron el Doctorado en Derecho, entre 2006 y 2011, totalizando aproximadamente 165 doctorandos. En el caso del Doctorado en Educación, se trató de 10 cohortes entre 2006 y 2014, con un total de 205 doctorandos y con la particularidad de que no todos eran brasileños. En una de las cohortes del año 2013 se sumaron 5 estudiantes provenientes de Angola, de algunas cohortes anteriores participaron también doctorandos mexicanos y ecuatorianos; pero en todos los casos, llegaron a través de ESJUS y vía Brasil.

La ciudad de Santa Fe, sede de la UCSF, es una ciudad pequeña de alrededor de 500.000 habitantes. La presencia de las cohortes de estudiantes de Brasil era notoria y los medios locales daban cuenta de ello. Los primeros grupos, provenientes del norte de Brasil tenían mayores dificultades idiomáticas, porque si bien se esforzaban por hablar en español, su acento del norte de Brasil era muy cerrado y esto dificultaba la comunicación. En las últimas cohortes, los doctorandos eran del sur de Brasil y las diferencias socio culturales se vieron modificadas considerablemente, encontrábamos una mayor afinidad.

La UCSF, adaptó el régimen de cursado de las carreras, de modo de favorecer la presencia de estudiantes extranjeros. Se diseñó un sistema de cursado intensivo con dos módulos quincenales durante dos años. Se cursaba quince días en el mes de febrero y otros quince en agosto. De esta 
manera, los costos del traslado se hacían menos onerosos para los doctorandos, y la modalidad intensiva del cursado durante toda la jornada, permitía optimizar el tiempo a los estudiantes extranjeros. A su vez, acentuaba los lazos entre ellos y de ellos con la Universidad en cuyas instalaciones permanecían de lunes a sábado.

c. Discusión y análisis de los resultados de la consulta directa

Con la finalidad de evaluar la experiencia atendiendo a las subjetividades de los estudiantes que participaron de ella, diseñamos -como ya hemos mencionado- una encuesta, Si bien se envió a todos los doctorandos, hemos recibido un número insuficiente de respuestas como para hacer generalizaciones. Aun así, es interesante notar que, entre los 61 doctorandos que hicieron su devolución, hay coincidencias importantes.

El trabajo de Corbella y Elías (2018), en el que analizan la problemática de los estudiantes extranjeros en la Universidad Nacional del Sur (UNS) en Argentina y proponen una serie de variables para establecer las razones por las cuales esos estudiantes eligen este país como destino para cursar sus estudios de grado, ha sido una referencia para analizar el caso de los estudiantes extranjeros de posgrado en la UCSF, si bien las autores trabajaron con una población diferente ya que indican que en su estudio"[...] se tomaron en cuenta los alumnos internacionales de grado, es decir, aquéllos que se movilizaron con la intención de cursar, complementar o concluir sus estudios en ese nivel". (Corbella y Elías, 2018, p. 130)

Las primeras preguntas estuvieron destinadas a recabar datos de los encuestados, tales como el año de inicio de la carrera en la UCSF y el país de residencia. Ello nos permitió tener una aproximación necesaria para poder ponderar las respuestas. De las cohortes 2007, 2008, 2009 y 2013 obtuvimos un mayor número de respuestas.
Los datos indicaron el $98 \%$ de las respuestas provenían de residentes en Brasil y el $2 \%$ restante de angoleños.

La tercera pregunta fue acerca de las razones que los motivaron a elegir a la Argentina como lugar de estudios de posgrado y se trató de una consigna de opción múltiple ${ }^{3}$. Un $82 \%$ de los encuestados (un total de 50 personas) resaltó la calidad académica de la educación de posgrado en Argentina, 16 respuestas que conforman el 26\% indicaron motivos económicos, específicamente el costo accesible de los estudios en el país; y la proximidad geográfica fue el tercer indicador en orden de importancia con un 19\% (Ver gráfico $\mathrm{N}^{\circ} 3$ )

Consultados en relación a las razones por las que eligió a la Universidad Católica de Santa Fe como lugar de estudio, 31 de los doctorandos se decidieron a partir de la recomendación de alguna persona de su confianza, esto es el 50,8\%; 27 lo hicieron por recomendación de la Consultora que ofició de intermediaria, es decir el 44\%; 6 indicaron como motivo la elección personal, totalizando el $9,8 \%$ y 1 encuestado se pronunció por otras razones, el 1,6\%, (Ver gráfico $\mathrm{N}^{\circ} 4$ )

Sin embargo, es importante aclarar que las cohortes puras de estudiantes extranjeros se organizaron a través de los convenios firmados con agencias como IFI y ENJUS, es decir que ninguno de ellos tramitó su matriculación directamente en la Universidad.

Las preguntas vinculadas a si recibieron ayuda económica y de qué tipo en caso de haberla recibido, nos proporcionaron los siguientes datos: tan solo 7 de los encuestados afirman haber recibido algún tipo de ayuda económica, lo que representa el $11 \%$ apenas. Dos recibieron ayudas económicas que les permitieron cubrir el costo total, y 5 recibieron ayudas económicas parciales. (Ver gráficos $\mathrm{N}^{\circ} 5$ y 6 )

\footnotetext{
${ }^{3}$ Esto hace que los porcentajes sumen por encima de 100.
} 
La pregunta siguiente estuvo vinculada a la trayectoria en la UCSF, y se les consultó acerca de si habían completado la carrera. El $52 \%$ manifestó haber completado el cursado, pero sin defender aún la tesis, el $27 \%$ completó la carrera y defendió la tesis y el $21 \%$ no completó el cursado.

La pregunta siguiente estuvo vinculada a la trayectoria en la UCSF, y se les consultó acerca de si habían completado la carrera. El 52\% manifestó haber completado el cursado, pero sin defender aún la tesis, el $27 \%$ completó la carrera y defendió la tesis y el $21 \%$ no completó el cursado.

Preguntados acerca de si continuaron sus estudios de posgrado en otra institución y pudieron doctorarse, obtuvimos 33 respuestas, de las cuales, 16 manifiestan estar escribiendo $\mathrm{su}$ tesis o tenerla presentada en proceso de evaluación y tan sólo cuatro, hacen referencia a que obtuvieron su doctorado en otras universidades.

Finalmente, se les inquirió acerca de la calidad de la experiencia como estudiantes de posgrado en Argentina, particularmente en los aspectos relacionados con la trayectoria académica y el nivel de satisfacción con la experiencia, como también a las dificultades que se les presentaron. Por tratarse de una consigna abierta resulta muy difícil tabular respuestas, pero los estudiantes destacan ampliamente la calidad y formación académica de los profesores (35 menciones), la calidad educativa de la UCSF (si bien se preguntó sobre la experiencia en Argentina, todas las respuestas estuvieron referidas a la UCSF), en menor medida aparecen mencionadas la calidad de las instalaciones de la casa de estudios y la experiencia de cursar en otro país y la "maravillosa experiencia que ello significó. Esta variable nos interesa especialmente ya que proporciona una aproximación a la percepción de los propios actores de ese proceso tan particular que conformaron estas cohortes en el marco de la Casa de Estudios, tanto desde el punto de vista académico como del de la gestión a través de agencias.

Además, la encuesta que aplicamos indaga en torno al recorrido de los estudiantes de posgrado en la UCSF, y a sus expectativas en orden a completar la carrera. Un total de 16 estudiantes, es decir el $26 \%$ terminó su doctorado y defendió su tesis; el $54 \%$ cursó y aprobó los seminarios, pero no presentó su tesis doctoral, se trata de 33 doctorandos, y 12 , es decir el $20 \%$, no completó el cursado. (Ver gráfico $\mathrm{N}^{\circ} 7$ )

De todas las personas que manifiestan haber completado el cursado, pero no han defendido la tesis en la UCSF, 4 manifiestan haberlo terminado en diferentes universidades de su país de origen (Univali, Universidad de Sao Paulo, Pontificia Universidad Católica de Brasil, Universidad de San Francisco Brasil). 11 dicen haber desestimado la posibilidad de obtener el Doctorado y 18, consultan sobre posibilidades para completarlo fuera de término en la UCSF. (Ver gráfico $\mathrm{N}^{\circ}$ 8). El resto se encuentra trabajando en la elaboración de su tesis o bien aguardando el dictamen y la posibilidad de su defensa.

Consultados sobre los aspectos positivos de la experiencia de haber sido estudiante de posgrado en Argentina, el $72 \%$ destaca la calidad del cuerpo docente y del programa de estudios de los doctorados de Derecho y Educación, así como la excelencia académica de la Universidad. También, aunque en menor medida, se referencia a la estructura edilicia, académica y de gestión de la Casa de Estudios y al clima acogedor hacia estos recién llegados.

Si bien la pregunta se requería la ponderación de su experiencia en Argentina, las respuestas están claramente asociadas a la experiencia en la Universidad.

Es interesante observar que un porcentaje de alrededor del 30\% destacaron la calidad educativa de la Universidad y un $72 \%$ a excelencia académica de sus profesores, pero no 
como razones de la elección. Sí consideraron que estos indicadores son motivo para recomendar la UCSF.

En cuanto a los aspectos negativos, los principales de acuerdo con lo manifestado fueron las demoras y dificultades en las comunicaciones y los inconvenientes surgidos de la gestión de las consultoras que actuaron como intermediarias. En total, el $44 \%$ señala la falta de fluidez en la comunicación y resolución de consultas y trámites, situación vinculada estrechamente a la intermediación de las agencias. Con relación al ámbito académico, la mayor dificultad estuvo en conseguir director de tesis, manifestaron 6 de los 63 encuestados, es decir, casi un 10\%.

Las consideraciones sobre rasgos positivos y negativos son muy interesantes para evaluar la experiencia, Un primer dato que nos proporcionan es que en general hay una alta satisfacción con la propuesta académica y su desarrollo; y en cambio, el descontento aumenta en relación con cuestiones administrativas y de gestión, situación que resultaba compleja en parte por la distancia y en parte por la intermediación de las consultoras. Sin embargo, en términos de la formación y de la oferta académica, es importante el porcentaje de doctorandos que las rescata como positivas.

\section{Conclusiones: el saldo de una experiencia}

El aumento de la movilidad académica internacional ha cobrado una dimensión muy importante en lo que va del presente siglo, acentuándose las migraciones Sur-Sur. En este contexto, el caso que describimos presenta una serie de particularidades que lo hacen muy interesante.

Este artículo constituye una primera aproximación al estudio de la movilidad académica internacional en la UCSF, un campo aún no abordado en esta Casa de Estudios y escasamente desarrollado en relación con las universidades santafesinas.
En primer lugar, debemos decir que es una experiencia que no se encuadra sin más en el concepto de migración ya que no se trató de estudiantes que se radicaran temporalmente en Santa Fe, sino que tan sólo permanecieron en la ciudad en cuatro ocasiones durante períodos no mayores a los veinte días. Tampoco puede encuadrarse la experiencia de las cohortes cerradas de extranjeros en la UCSF como un caso de internacionalización académica en sentido estricto. El concepto, como hemos explicitado, refiere a las políticas universitarias de cooperación e intercambio en un marco más amplio que la migración de estudiantes.

Por ello proponemos el concepto de movilidad académica internacional como el más adecuado y preciso para describir esta experiencia. Esta categoría nos permite abordar la dimensión de las decisiones institucionales y los vínculos interinstitucionales (en este caso, entre la UCSF y las consultoras), y a la vez, las subjetividades expresadas en las valoraciones de los doctorandos acerca de su experiencia.

Hay que decir entonces que, atendiendo a las subjetividades, estos doctorandos extranjeros se encontraron con algunos de los mismos desafíos de los estudiantes que migran por algunos años para cursar sus estudios de grado o, en menor medida, de posgrado; como pueden ser las dificultades con el idioma, costos de alojamiento y comida y la necesidad de adaptarse a pautas culturales más o menos diferentes a las propias según los casos. De todos modos, las estadías cortas en Santa Fe minimizaron esas dificultades, aunque posiblemente incrementaron otras vinculadas a la distancia.

Con relación a las decisiones institucionales y los acuerdos interinstitucionales, retomamos los aportes de García (2016) acerca del rol del mercado en este nuevo escenario educativo que se inauguró a fines del siglo XX y que marca el ritmo del surgimiento y expansión de los estudios de posgrado, así como las posibilidades de su 
financiamiento a través de aranceles. Se explica en parte, a partir de este escenario, la decisión de la UCSF de ofrecer sus propuestas académicas a cohortes cerradas de estudiantes extranjeros, decisión enmarcada en los convenios firmados con entidades como IFI y ENJUS que hacían las veces de intermediarias entre los interesados y las ofertas académicas de varias universidades argentinas.

La movilidad académica sur-sur de las cohortes provenientes de Brasil, exigió adecuar estructuras para hacer factible las trayectorias académicas de estos migrantes, así por ejemplo, se implementó una modificación del formato para el dictado de los Seminarios, comprimiendo el dictado de clases en jornadas intensivas en dos períodos de quince días durante dos años, para favorecer el cursado presencial de las cohortes de extranjeros que podían trasladarse por períodos cortos sin abandonar sus trabajos y actividades. En la experiencia que narramos los estudiantes llegaron a Santa Fe vía Brasil, aun cuando no todos eran brasileños. Además de dos doctorandos de origen mexicano y dos de Ecuador, una de las cohortes del año 2013 contó con un subgrupo de angoleños que fueron incentivados por las autoridades de su país para cursar sus estudios de posgrado en Argentina.

De las encuestas que aplicamos a los doctorandos, se desprende una valoración positiva de la oferta académica de la UCSF y una ponderación favorable a la cordialidad de profesores y personal de la Casa, pero también opiniones menos favorables en relación a cuestiones vinculadas al campo administrativo y de gestión académica; situación que resulta explicable si se tiene en cuenta la intermediación de las consultoras y la distancia geográfica que no siempre es posible suplir eficazmente con medios digitales. El hecho de que los estudiantes no se radicaran en Santa Fe y desconocieran la dinámica del sistema universitario argentino en buena medida, sumado a la escasa frecuencia de sus viajes a este país, fueron otros factores que permiten comprender las dificultades en trámites $\mathrm{y}$ gestiones.

Para la UCSF, el saldo de la experiencia ha sido positivo. Ha sentado precedente para otras cohortes cerradas de carreras de grado y de posgrado, ha permitido visualizar una serie de dificultades de orden administrativo y de gestión, así como comunicacionales que constituyen un desafío para alcanzar mayor fluidez en la resolución de las cuestiones vinculadas a estas áreas; y por último ha definido para los doctorados una modalidad de cursado intensivo que se mantiene y posibilita la presencia en actuales cohortes de doctorandos extranjeros y de otros que provienen de distintas regiones de la Argentina.

En cuanto a la baja proporción de doctorandos que han defendido su tesis, es un asunto que amerita otro estudio, ya que no se trata de un fenómeno privativo de estas cohortes y ni siquiera de esta Universidad. Sin embargo, debe considerarse también la situación de un porcentaje nada despreciable de doctorandos que se encuentran aguardando su defensa de tesis, la evaluación de estas o en el proceso de su escritura.

Para cerrar este escrito, diremos que la experiencia de las cohortes cerradas provenientes de Brasil en los Doctorados de Educación y Derecho de la UCSF, pueden considerarse un caso muy particular de migración Sur - Sur: una migración por pertenencia académica. Y que constituye una valiosa experiencia en orden a pensar acciones futuras y a generar vinculaciones académicas más complejas con otras instituciones de América del Sur.

\section{Referencias}

Abba, M. (2019) La internacionalización de la educación superior como un proceso dialectico.

Percepciones, concepciones y prácticas en las universidades. EDUCO, Neuquén. pp. 79-94. 
Cardozo, G. (2015). Estudiantes extranjeros/as de la UNLa. XI Jornadas de Sociología. Facultad de Ciencias Sociales, Universidad de Buenos Aires, Buenos Aires.

CONEAU. (2019). La CONEAU y el sistema de acreditación regional ARCU-SUR; editado por Jorge Lafforgue; Laura Romero. - 1a ed. - Ciudad Autónoma de Buenos Aires. CONEAU - Comisión Nacional de Evaluación y Acreditación Universitaria.

Corbella, V. y Elías, S. (2018) Movilidad estudiantil universitaria: ¿qué factores inciden 'en la decisión de elegir Argentina como destino?. Perfiles educativos, vol. XL, núm. 160. UNAM, México. pp. 120- 140.

García, L. (coord). (2016). Situación actual del posgrado en Argentina, Brasil y Paraguay: carreras, estudiantes y egresados. Nora Z. Lamfri (coord. gral). Los posgrados en Argentina, Brasil y Paraguay: aproximaciones comparadas en contextos de evaluación de la calidad de la educación superior - 1a ed. Córdoba: Encuentro Grupo Editor. P. 75-145.

Lamfri, N. y Salto, D. (2016). La internacionalización de los posgrados en la región. Los casos de Argentina, Brasil y Paraguay. Nora Z. Lamfri (coord. gral). Los posgrados en Argentina, Brasil y Paraguay: aproximaciones comparadas en contextos de evaluación de la calidad de la educación superior - 1a ed. - Córdoba: Encuentro Grupo Editor. pp $214-248$.

Lvovich, D. (2009). Resultados e impactos de los programas de apoyo a la formación de posgrado en Argentina. Revista Iberoamericana de Ciencia, Tecnología y Sociedad - CTS, vol. 5, núm. 13, noviembre, 2009- pp. 157-173. Centro de Estudios sobre Ciencia, Desarrollo y Educación Superior
Buenos Aires. Argentina.

Mayer, L. y Catalano, B. (2018) Internacionalización de la educación y movilidad: reflexiones a partir del caso argentino. Universitas. Revista de Ciencias Sociales y Humanas, $\mathrm{N}^{\circ} 29$. pp. 19 -41.

Passarini, J.; Zucarelli, V. y De León, F. (2018). Evaluación de las movilidades de los estudiantes como Aporte a la mejora de la internacionalización. Actas del $\mathrm{V}$ Congreso Internacional "La Gestión Estratégica de la Calidad en las IES de América Latina y el Caribe ante los desafíos del Desarrollo Sostenible". pp. $115-127$.

Ramirez Valdivia, M. y Latorre Bahamondez, P. (2019). Conceptos generales sobre internacionalización universitaria. Internacionalización e integración regional. Percepciones, concepciones y prácticas en las universidades. EDUCO, Neuquén. Pp 35- 58.

Sangiácomo, M. (2002). Migrar para estudiar. Los estudiantes bolivianos en la Universidad Nacional de La Plata [en línea]. Sociohistórica, (11-12). Disponible en: http://www.fuentesmemoria.fahce.unlp.edu. ar/art_revistas/pr.3068/pr.

Sosa, M. (2016). Migrantes en el sistema educativo argentino. Un estudio sobre la presencia de alumnos extranjeros en los estudios de nivel superior. RIES universia.net, $\mathrm{N}^{\circ}$ 19, Vol. VII. pp. 97- 116.

UCSF. (2014). Modelo Educativo. Santa Fe, Editorial UCSF.

UNESCO. (2013). Situación Educativa de América Latina y el Caribe: Hacia la educación de calidad para todos al 2015. Publicado en 2013 por la Oficina Regional de Educación para América Latina y el Caribe (OREALC/UNESCO Santiago). 


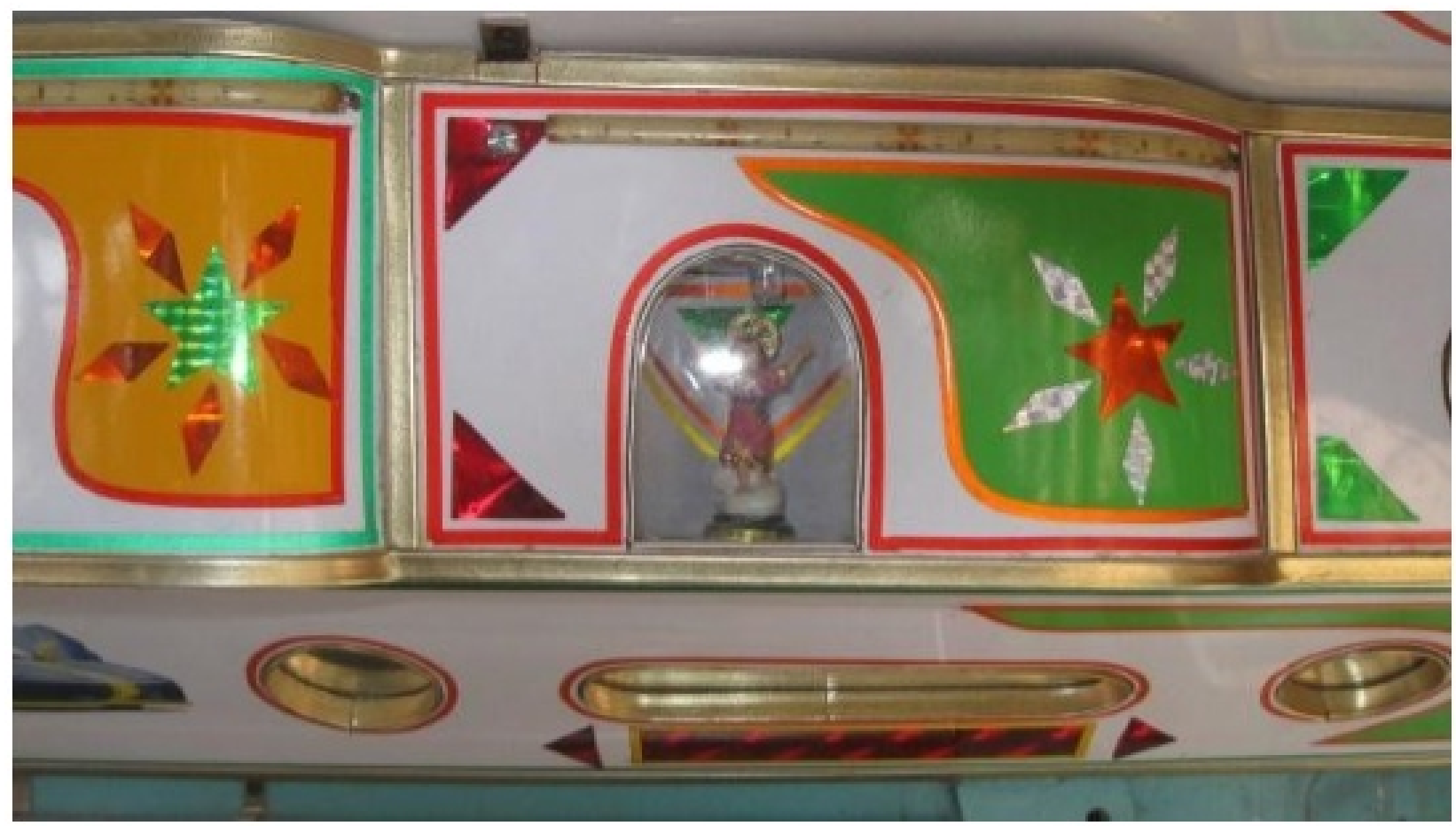

Número de la obra: 84

Título: Chiva "El Lucerito"

Autora: Margarita Chávarro Castro

Lugar: Municipio La Plata, Huila

Fecha de captura: Abril de 2013

Técnica: Fotografía digital

Fuente: Colección Margarita Chávarro Castro 